Francis R. Verdun

Daniel Gutierrez

John Paul Vader

Abbas Aroua

Leonor Trinidad Alamo-Maestre

François Bochud

François Gudinchet

\title{
CT radiation dose in children: a survey to establish age-based diagnostic reference levels in Switzerland
}

\author{
L. T. Alamo-Maestre · F. Gudinchet \\ University Hospital of Lausanne (CHUV), \\ Department of Radiodiagnostic \\ and Interventional Radiology, \\ CHUV and University of Lausanne, \\ 1011 Lausanne, Switzerland
}

\begin{abstract}
This work aimed at assessing the doses delivered in Switzerland to paediatric patients during computed tomography (CT) examinations of the brain, chest and abdomen, and at establishing diagnostic reference levels (DRLs) for various age groups. Forms were sent to the ten centres performing $\mathrm{CT}$ on children, addressing the demographics, the indication and the scanning parameters: number of series, kilovoltage, tube
\end{abstract}

current, rotation time, reconstruction slice thickness and pitch, volume $\mathrm{CT}$ dose index $\left(\mathrm{CTDI}_{\mathrm{vol}}\right)$ and dose length product (DLP). Per age group, the proposed DRLs for brain, chest and abdomen are, respectively, in terms of $\mathrm{CTDI}_{\mathrm{vol}}$ : 20, 30, 40, $60 \mathrm{mGy}$; 5, 8, 10, $12 \mathrm{mGy} ; 7,9,13,16 \mathrm{mGy}$; and in terms of DLP: 270, 420, 560, $1,000 \mathrm{mGy} \mathrm{cm;} \mathrm{110,} \mathrm{200,} \mathrm{220,}$ 460 mGy cm; 130, 300, 380, 500 mGy $\mathrm{cm}$. An optimisation process should be initiated to reduce the spread in dose recorded in this study. A major element of this process should be the use of DRLs.

Keywords Computed tomography · Paediatrics - Radiation dosimetry · Radiation protection .

Diagnostic reference levels

\section{Introduction}

Since its first applications in the early 1970s the use of computed tomography (CT) has been continuously growing. According to the 2000 report [1] of the United Nations Scientific Committee on the Effects of Atomic Radiation (UNSCEAR), the frequency of CT examinations in countries of high healthcare level increased on average from 6.1 per year per 1,000 population in the 1970 s to 48 per year per 1,000 population in the period 1991-1996, while the average effective dose per CT examination increased from $1.3 \mathrm{mSv}$ in the $1970 \mathrm{~s}$ to $8.8 \mathrm{mSv}$ in the period 1991-1996. This increase went on steadily during the last decade. In Switzerland, the results of the 2003 survey on the exposure of the population by medical X-ray imaging [2] indicated a 70\% increase of CT examinations in a 5-year period (1998-2003), which, combined with a $20 \%$ increase in the average effective dose per CT procedure, led to an increase by a factor of two in the CT contribution to the collective dose due to medical X-rays reaching $47 \%$. The same trend was registered in other European countries. In Norway, the frequency of $\mathrm{CT}$ examinations increased by a factor of 2.2 in a decade and CT contribution to collective effective dose was estimated to account for $59 \%$ of the total, as opposed to $30 \%$ in the previous survey [3]. In Germany, the increase in the collective dose due to CT rose by about $50 \%$ between 1996 and 2002, and in the UK the frequency of CT examinations increased by $39 \%$ between 1998 and 2002. 
The proportion of all CT examinations which are performed in children does not exceed a few per cent. In Switzerland, the most recent nationwide survey on the exposure of the population by diagnostic and interventional radiology that provided patient age data indicated a CT paediatric fraction of $1 \%$ in $1998[4,5]$. The same figure was reported in Germany for the period 2005-2006 [6]. Other countries reported higher values. Recent surveys covering the year 2000 revealed a paediatric fraction of $2.7 \%$ in Japan [7], and of $6.5 \%$ in the USA [8]. The latter figure is comparable with that given by UNSCEAR for the period 1991-1996 [1]. Only general guidelines are available for the use of $\mathrm{CT}$ in the paediatric population [9-13] and the need for size-based CT protocols has only recently been emphasised [6, 14-18]. Given the recent attention to radiation risks and CT in children [19-24] there is an urgent need for optimisation of the present practice by means of the introduction of the Diagnostic Reference Level (DRL) by the International Commission on Radiological Protection (ICRP) in 1997 [25]. For these reasons, we sought to assess the practice of paediatric CT in Switzerland using forms sent to the ten main centres (including regional and university hospitals) that perform CT examinations in children. The results of this survey allow the development of a set of DRL values for the most common paediatric CT examinations.

\section{Materials and method}

The survey conducted between January and December 2005 consisted of a questionnaire addressed to the ten centres dealing with paediatric CT in Switzerland (Aarau, Bellinzona, Bern, Fribourg, Geneva, Lausanne, St Gallen, Sion, Winterthur, and Zurich). The data were treated in an anonymous way, with the ten centres being coded by alphabetical letters from $\mathrm{A}$ to $\mathrm{J}$.
In this survey, the paediatric population was separated into four age/weight groups ( $<1$ year or $<10 \mathrm{~kg} ; 1-5$ years or $10-20 \mathrm{~kg} ; 5-10$ years or $20-35 \mathrm{~kg} ; 10-15$ years or $>35 \mathrm{~kg}$ ). Each centre was asked to give the total number per age group of children undergoing CT of the brain, chest and abdomen during the year 2005. The generic indications of the examinations were chosen to be compatible with a previous survey conducted in the UK in 2003 [26]: trauma for brain and detection of malignancy for chest. The indication for the CT of the abdomen, which was not considered in the UK survey, was detection of malignancy.

For each type of examination and each age group, the type of CT machine and the CT parameters - including the number of series, the use of a contrast medium, the tube kilovoltage and current, the rotation time, the reconstruction slice thickness, the pitch, the displayed volume CT dose index $\left(\mathrm{CTDI}_{\mathrm{vol}}\right)$ and the dose length product (DLP) as defined according to [27] - were requested. No dose measurements were carried out in the participating centres, but the readings of all the $\mathrm{CT}$ systems operating in Switzerland are periodically checked by the inspectors of the Federal Office of Public Health and the tolerated uncertainty according to the law is $\pm 30 \%$. A consistency check of the CTDI $\mathrm{vol}_{\mathrm{vo}}$ and DLPs collected was performed using the dosimetric characteristics of the CT machines used by means of the data published on the Impact Scan website [28]. At the end of the survey each centre received a final report to verify if the data recorded was correct and if some comment needed to be added.

Since no data related to CT examination performed on specific patients was collected, the DRL for a type of examination (and the associated indication) and for an age group is not established directly by taking the 75-percentile of a dose distribution, but indirectly based on the mean value of the dosimetric quantity $\left(\mathrm{CTDI}_{\mathrm{vol}}\right.$ or DLP). In this alternative method, already used in the past in situations where no dose distributions were available $[29,30]$, the

Table 1 Characteristics of the CT units used

\begin{tabular}{llll}
\hline Centre & Manufacturer & Model & Type \\
\hline $\mathrm{A}^{\mathrm{a}}$ & Siemens & Sensation 16 (Body examinations) & MSCT (16-slice system) \\
& GE & LightSpeed 16 (Brain examinations) & MSCT (16-slice system) \\
B & Philips & Tomoscan SR 7000 SSCT & SSCT \\
C & Philips & Mx 8000 IDT (16 slices) & MSCT (16-slice system) \\
$\mathrm{D}^{\mathrm{a}}$ & Philips & Mx 8000 IDT (16 slices) & MSCT (16-slice system) \\
E & GE & LightSpeed 8 & MSCT (8-slice system) \\
$\mathrm{F}$ & Siemens & Sensation 64 & MSCT (64-slice system) \\
$\mathrm{G}^{\mathrm{a}}$ & GE & LightSpeed 16 & MSCT (16-slice system) \\
$\mathrm{H}$ & Toshiba & Aquilion 16 & MSCT (16-slice system) \\
$\mathrm{I}^{\mathrm{a}}$ & Picker & PQ 5000 & SSCT \\
$\mathrm{J}$ & GE & CT/i PRO & SSCT \\
\hline
\end{tabular}

${ }^{\mathrm{a}}$ University or paediatric hospital 
Table 2 Number of examinations performed in the various centres

\begin{tabular}{lllll}
\hline Centre & $\begin{array}{l}0-5 \text { years } \\
(0-20 \mathrm{~kg})\end{array}$ & $\begin{array}{l}5-10 \text { years } \\
(20-35 \mathrm{~kg})\end{array}$ & $\begin{array}{l}10-15 \text { years } \\
(>35 \mathrm{~kg})\end{array}$ & Total \\
\hline $\mathrm{A}^{\mathrm{a}}$ & 208 & 126 & 186 & 520 \\
$\mathrm{~B}$ & $10^{\mathrm{b}}$ & $7^{\mathrm{b}}$ & $8^{\mathrm{b}}$ & 25 \\
$\mathrm{D}^{\mathrm{a}}$ & 304 & 219 & 290 & 813 \\
$\mathrm{E}$ & 29 & 35 & 33 & 97 \\
$\mathrm{~F}$ & 69 & 76 & 145 & 290 \\
$\mathrm{G}^{\mathrm{a}}$ & 261 & 212 & 217 & 690 \\
$\mathrm{I}^{\mathrm{a}}$ & 563 & 273 & 203 & 1039 \\
$\mathrm{~J}$ & 26 & 38 & 86 & 150 \\
$\mathrm{All}$ & 1,470 & 986 & 1,168 & 3,624 \\
\hline
\end{tabular}

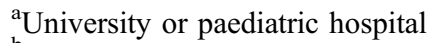

${ }^{\mathrm{b}}$ Based on the total number and using the average distribution of all the other centres

DRL is obtained by multiplying the mean value, obtained by averaging the typical values provided by the participating centres, by an appropriate factor (1.25 in the present work). Due to the typical shape of the dose distributions, this method provides a figure comparable with the third quartile. Each centre provided for each anatomical region and clinical indication typical values for the various age groups, taking into account the variability due to differences in the protocols used (number of sequences, technical parameters, etc.). Therefore, the average values over the participating centres expressed the national variability for a given examination/indication.

\section{Results}

Information was obtained from eight out of the ten centres. Two centres $(\mathrm{C}$ and $\mathrm{H})$, which are small regional hospitals, could not provide it due to the limitation of their accounting systems. Three centres were not able to provide detailed information and gave either the number of all examinations ventilated by age, but merging the first two age categories $(<1$ year and 1-5 years) into a single one ( $0-5$ years), (centres $\mathrm{D}$ and $\mathrm{F}$ ), or the total number of examinations (centre B). The CT systems involved in the survey are presented in Table 1.

The total frequencies of all types of examinations are given in Table 2. The data obtained from centres A, G and $\mathrm{J}$ specific to the two age categories $(<1$ year and $1-5$ years $)$ were summed up into a single one (0-5 years), in order to allow the comparison with the other centres. Moreover, in the case of centre B, the data presented for the three age groups was calculated based on the total number of examinations and using the average distribution of all the other centres. Table 3 presents the frequency data ventilated by type of examination, associated with the five centres that provided such detailed information (A, E, G, I and J).

Table 4 displays the dose results of the survey concerning the CT examinations of the brain, the chest and the abdomen and for the four age groups. Mean values averaged over the participating centres and ranges of the $\mathrm{CTDI}_{\mathrm{vol}}(\mathrm{mGy})$ and the DLP (mGy $\left.\mathrm{cm}\right)$ values are given. Table 5 gives the DRL values, in terms of the $\mathrm{CTDI}_{\mathrm{vol}}$ (mGy) and the DLP (mGy $\mathrm{cm})$, established as described above for the three types of CT examinations and the four age groups, as well as the corresponding DRLs reported in the literature for Germany and the UK.

Table 3 Number of examinations per type of examination. (Only the five centres, A, E, G, I and J, which gave detailed information, are considered)

\begin{tabular}{lllll}
\hline Examination & $\begin{array}{l}0-5 \text { years } \\
(0-20 \mathrm{~kg})\end{array}$ & $\begin{array}{l}5-10 \text { years } \\
(20-35 \mathrm{~kg})\end{array}$ & $\begin{array}{l}10-15 \text { years } \\
(>35 \mathrm{~kg})\end{array}$ & Total \\
\hline Brain & 793 & 407 & 351 & 1,551 \\
Chest & 173 & 146 & 198 & 517 \\
Abdomen & 121 & 131 & 176 & 428 \\
Total & 1,087 & 684 & 725 & 2,496 \\
\hline
\end{tabular}


Table 4 Means values averaged over participating centres and ranges of $\mathrm{CTDI}_{\mathrm{vol}}(\mathrm{mGy})$ and the DLP (mGy $\left.\mathrm{cm}\right)$ values

\begin{tabular}{|c|c|c|c|c|c|c|c|c|c|c|}
\hline \multirow[t]{2}{*}{ Age group } & \multirow[t]{2}{*}{ Quantity } & \multicolumn{3}{|c|}{ Brain } & \multicolumn{3}{|c|}{ Chest } & \multicolumn{3}{|c|}{ Abdomen } \\
\hline & & $n$ & Mean & Range & $n$ & Mean & Range & $n$ & Mean & Range \\
\hline \multirow{2}{*}{$<1$ year } & $\mathrm{CTDI}_{\mathrm{vol}}$ & 9 & 17 & $8.3-41$ & 9 & 4.2 & $0.8-11$ & 8 & 5.9 & $1.4-16$ \\
\hline & DLP & 9 & 213 & $66-414$ & 9 & 85 & $12-198$ & 8 & 107 & $29-258$ \\
\hline \multirow[t]{2}{*}{$1-5$ years } & $\mathrm{CTDI}_{\mathrm{vol}}$ & 10 & 25 & $13-41$ & 10 & 6.5 & $0.8-20$ & 10 & 7.2 & $1.4-16$ \\
\hline & DLP & 10 & 332 & $144-538$ & 10 & 161 & $12-617$ & 10 & 238 & $29-650$ \\
\hline \multirow[t]{2}{*}{$5-10$ years } & $\mathrm{CTDI}_{\mathrm{vol}}$ & 10 & 32 & $13-50$ & 10 & 7.6 & $2.1-20$ & 10 & 10 & $1.4-17$ \\
\hline & DLP & 10 & 451 & $173-621$ & 10 & 178 & $32-352$ & 10 & 308 & $29-627$ \\
\hline \multirow[t]{2}{*}{$10-15$ years } & $\mathrm{CTDI}_{\mathrm{vol}}$ & 10 & 45 & $14-85$ & 10 & 9.6 & $2.1-26$ & 10 & 13 & $4.5-18$ \\
\hline & DLP & 10 & 805 & $216-1^{\prime} 485$ & 10 & 366 & $32-1^{\prime} 298$ & 10 & 398 & $90-738$ \\
\hline
\end{tabular}

As an example of the dose distribution involved, Fig. 1 summarises the data obtained for children in the age group 1-5 years. It displays graphically the relation between the DLP and the CTDI $\mathrm{vol}_{\mathrm{vo}}$. The slope of the distribution of points expresses the anatomical length since, by definition, the DLP is the product of the CTDI ${ }_{\mathrm{vol}}$ and the length of the volume examined. This information is of particular importance as the actual length exposed to primary radiation tends to increase when switching from a SSCT to a MDCT as shown by [26]. Moreover, one needs to control the over-ranging of the examination which tends to increase with the size of the beam collimation of the CT unit.

\section{Discussion}

The participating centres that carry out paediatric $\mathrm{CT}$ in Switzerland use a wide range of CT technologies. Table 1 shows that most centres are using multiple-slice CT (MSCT): one eight-slice, five 16-slice and one 64-slice systems from the four main CT manufacturers (General
Electric, Philips, Siemens and Toshiba). However, three centres, one of which is a University centre (I) are still using single slice CT (SSCT) units. There is a general trend in Switzerland to replace SSCT by MSCT systems and this hold for the total number of $216 \mathrm{CT}$ in the country.

The eight centres that provided frequency data performed a total of 3,624 CT examinations in 2005 (Table 2), with an average of 453 per centre and a variability from 25 in a small centre (B) to 1,039 for a big centre (I). Forty-one per cent of the examinations are performed on newborns and children before the age of $5,27 \%$ on children between 5 and 10 , and $32 \%$ on children between 10 and 15 . Table 3 shows that brain examinations represent on average $62 \%$ of the total, whereas chest and abdomen account for $21 \%$ and $17 \%$, respectively. For the age category $0-5$ brain examination accounts for as high as $73 \%$. Three centres only (A, $\mathrm{G}$ and $\mathrm{J}$ ) provided data separately for the two age groups $<1$ and $1-5$, revealing that $16 \%$ of the examinations are performed on newborns and $84 \%$ on children between 1 and 5 .

Large variations in dose appear between the different centres (Table 4). These variations are reflected in Fig. 1

Table 5 DRL values in terms of $\mathrm{CTDI}_{\mathrm{vol}}(\mathrm{mGy})$ and the DLP $(\mathrm{mGy} \mathrm{cm})$ established in the present work compared with the data reported in Germany $(D)$ and the UK

\begin{tabular}{|c|c|c|c|c|c|c|c|c|c|c|}
\hline \multirow[t]{2}{*}{ Age group } & \multirow[t]{2}{*}{ Quantity } & \multicolumn{3}{|l|}{ Brain } & \multicolumn{3}{|l|}{ Chest } & \multicolumn{3}{|l|}{ Abdomen } \\
\hline & & This work & $\mathrm{D}[6]$ & UK [26] & This work & $\mathrm{D}[6]$ & UK [26] & This work & $\mathrm{D}[6]$ & UK [26] \\
\hline \multirow[t]{2}{*}{$<1$ year } & $\mathrm{CTDI}_{\mathrm{vol}}$ & 20 & 33 & 30 & 5 & 3.5 & 12 & 7 & 5 & $20^{\mathrm{a}}$ \\
\hline & DLP & 270 & 390 & 270 & 110 & 55 & 200 & 130 & 145 & $170^{\mathrm{a}}$ \\
\hline \multirow[t]{2}{*}{$1-5$ years } & $\mathrm{CTDI}_{\mathrm{vol}}$ & 30 & 40 & 45 & 8 & 5.5 & 13 & 9 & 8 & $20^{\mathrm{a}}$ \\
\hline & DLP & 420 & 520 & 470 & 200 & 110 & 230 & 300 & 255 & $250^{\mathrm{a}}$ \\
\hline \multirow[t]{2}{*}{$5-10$ years } & $\mathrm{CTDI}_{\mathrm{vol}}$ & 40 & 50 & 50 & 10 & 8.5 & 20 & 13 & 13 & $30^{\mathrm{a}}$ \\
\hline & DLP & 560 & 710 & 620 & 220 & 210 & 370 & 380 & 475 & $500^{\mathrm{a}}$ \\
\hline \multirow[t]{2}{*}{$10-15$ years } & $\mathrm{CTDI}_{\mathrm{vol}}$ & 60 & 60 & 65 & 12 & 6.8 & 14 & 16 & 10 & 14 \\
\hline & DLP & $1^{\prime} 000$ & 920 & 930 & 460 & 205 & 580 & 500 & 500 & 560 \\
\hline
\end{tabular}

${ }^{\mathrm{a}}$ These values recommended in the EU are taken from reference [31]. For the UK, adult values were taken for age group 10-15 years since they were not available in the report 

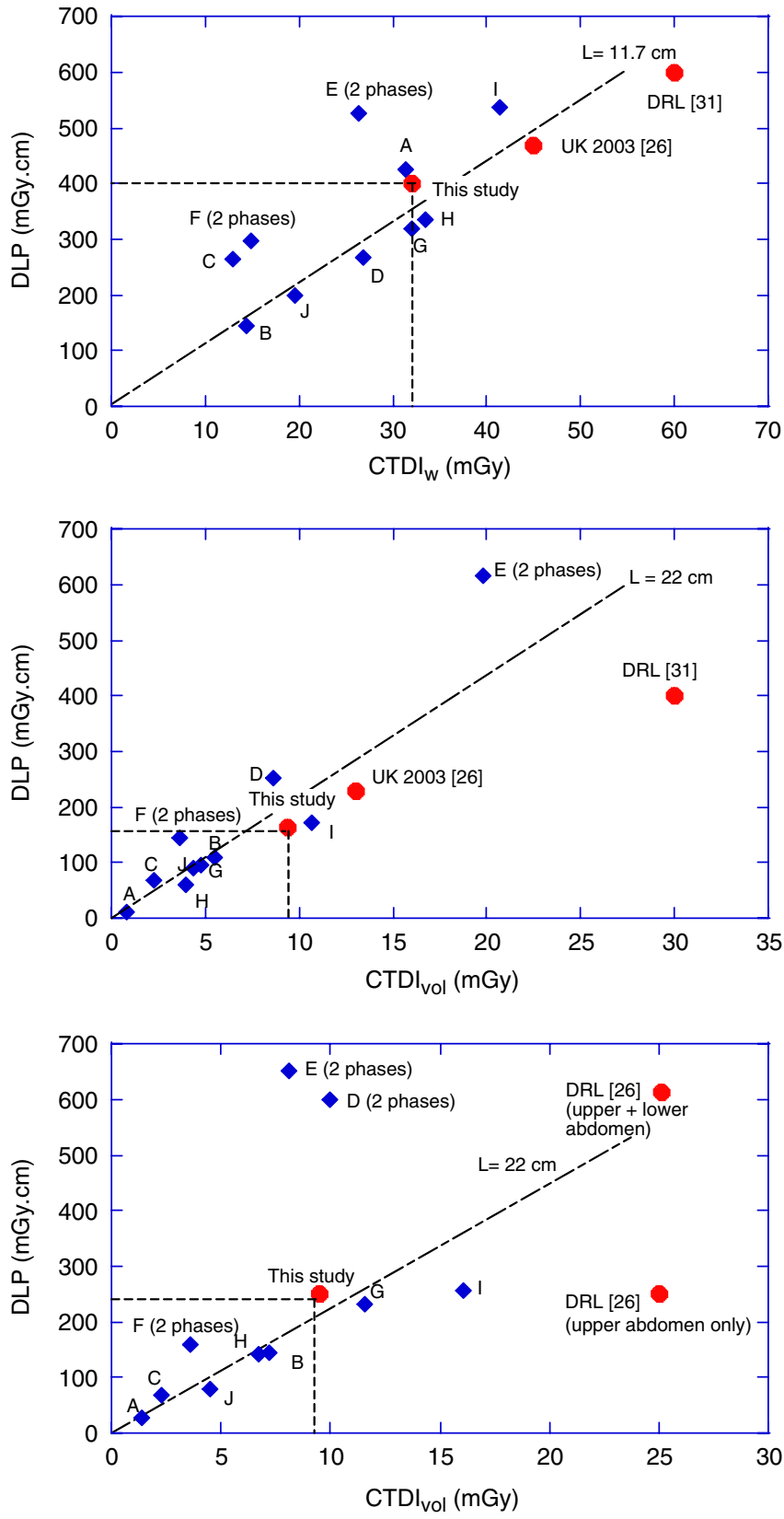

Fig. 1 Brain (top), chest (middle) and abdominal (bottom) examination for children in age group 1 to 5 years

that displays the plot of the DLP as a function of the $\mathrm{CTDI}_{\mathrm{vol}}$ and the DLP. For brain examination, almost all data points can be aligned along a straight line that has a slope of $11.7\left(R^{2}=0.86\right)$, meaning that the average length of coverage is $11.7 \mathrm{~cm}$ for this particular examination and age category. This examination length is fully compatible with the data obtained in the UK in $2003(10.9-11.7 \mathrm{~cm})$ [26]. For both chest and abdomen examinations, the slope is 22 $\left(R^{2}=0.94\right.$ and $R^{2}=0.97$, respectively), i.e. an average scan length of $22 \mathrm{~cm}$. It is of note that the value obtained for the chest in this survey for a similar age category is slightly higher than the UK data $(16.5-18.6 \mathrm{~cm})$ [26]. Table 4 shows that the inter-centre variation for brain examination and for the different age groups ranges from a factor of 3.26.2 for the $\mathrm{CTDI}_{\mathrm{vol}}(3.6-6.9$ for the DLP) the highest variation being for age group 10-15 years where the $\mathrm{CTDI}_{\mathrm{vol}}$ delivered varies from 14 to $85 \mathrm{mGy}$ and the DLP varies from 216 to $1,485 \mathrm{mGy} \mathrm{cm}$. For chest examination the inter-centre variations for the different age groups range from a factor of 9.5-25 for the $\mathrm{CTDI}_{\mathrm{vol}}$ (11-51.4 for the DLP), the highest variation being for age group 1-5 years where the $\mathrm{CTDI}_{\mathrm{vol}}$ delivered varies from 0.8 to $20 \mathrm{mGy}$ and the DLP varies from 12 to $617 \mathrm{mGy} \mathrm{cm}$. For abdomen examination, the inter-centre variations for the different age groups ranges from a factor of 4.0-12.1 for the CTDI ${ }_{\mathrm{vol}}$ (8.2-22.4 for the DLP), the highest variation being for age group 1-5 years where the $\mathrm{CTDI}_{\mathrm{vol}}$ delivered varies from 1.4 to $16 \mathrm{mGy}$ and the DLP varies from 29 to $650 \mathrm{mGy} \mathrm{cm}$.

It is worth mentioning that only one centre (I) that performs more than $1,000 \mathrm{CT}$ examinations with a SSCT system does not adapt the protocols to the age of the patient. Thus, there is great potential for optimisation. The small centres (E and F) recourse systematically to a biphase acquisition. This practice is not adopted by the other centres for brain and chest examinations. Centre D (large) performs also a bi-phase acquisition during abdomen examinations, which leads to high dose values. Here again there is a potential for optimisation by specifying accurately the instances for which bi-phase acquisition is indicated.

The great dispersion of the $\mathrm{CTDI}_{\mathrm{vol}}$ reflects a great dispersion of the associated image quality. The majority of the big centres produce images of homogeneous quality since they are well grouped. Centre I only, where no adaptation of protocols is used, the images produced are of excellent quality for a very young population of patients. On the other hand, in certain centres, where only a few examinations are carried out, such low CTDI values are used $(0.8 \mathrm{mGy}$ for chest and patients between 0 and 5 years; $1.4 \mathrm{mGy}$ for abdomen and patients from 0 and 10 years) that the diagnostic quality of the image may be questioned.

As shown in Table 5, the DRLs in terms of CTDI ${ }_{\mathrm{vol}}$ and DRLs are compatible with the values reported in Germany and the UK. In most cases they are lower than the UK data. Compared with the German data, the values established in this work are lower for the brain examination, but higher for chest and abdomen for some age groups.

The DRLs obtained in this investigation were established by considering typical dose values associated with standard protocols used in a given centre for the various types of examinations and age groups, and then averaging over the ten participating centres and multiplying by an appropriate factor. These DRLs should be considered as provisional only, to be replaced in the future by more robust data established through surveys of measured doses 
on a big sample of patients leading to empirical dose distributions. The collection of such data is time and resource consuming, but the new Swiss legislation concerning the implementation of DRLs and the settingup of a national dose database, as well as the standardisation of the way the dose descriptors $\left(\mathrm{CTDI}_{\mathrm{vol}}\right.$ and DLP parameters) will be stored in the DICOM header, which is under discussion, will all facilitate such an endeavour.

\section{Conclusion}

The frequency of paediatric CT examinations and the typical values of the related dose quantities $\left(\mathrm{CTDI}_{\mathrm{vol}}\right.$ and DRLs) were surveyed in the ten Swiss centres performing paediatric CT. Mean values averaged over the participating centres were calculated and the corresponding DRLs were established by multiplying the mean values by 1.25 . This investigation revealed that 4,000-5,000 CT examinations are carried out on children in Switzerland, with an average of 453 per centre performing paediatric CT. Significant variations of the radiation dose delivered to the paediatric popu- lation were found. An optimisation process should be initiated in order to reduce this spread in dose (appropriate image quality requirements for a given indication, number of acquisition phases that are clinically relevant, etc.). A major element of the optimisation process is a consensus on the DRLs that need to be used. This becomes a priority in the light of contributions such as described in a recent article published in the Lancet [32]. A set of DRL values for CT examinations of the brain, the chest and the abdomen and for the various age groups are proposed here for temporary use in paediatrics until a more extensive survey is organised to collect dose data on a large sample of patients and to establish empirical dose distributions.

Acknowledgements The authors gratefully acknowledge financial support from the Swiss National Science Foundation (Grant no. 3200B0-105951) and are thankful to the members of SSPR, in particular: Drs G. Eich, S. Hanquinet, J.-M. Girard, A. Racle, G. Remsei, T. Schraner, P. Waibel, R. Wolf, Mme M. Wyttenbach, M. P. Brégis, and A. Devaud)

\section{References}

1. United Nations Scientific Committee on the Effects of Atomic Radiation (2000) Report to the general assembly, annex D: Medical radiation exposures. United Nations, New York

2. Aroua A, Vader JP, Valley JF, Verdun FR (2007) Exposure of the Swiss population by radiodiagnostics : 2003 Review. Health Phys 92:442-448

3. Børretzen I, Lysdahl KB, Olerud HM (2007) Diagnostic radiology in Norway - trends in examination frequency and collective effective dose. Radiat Prot Dosim 124:339-347

4. Aroua A, Burnand B, Decka I, Vader JP, Valley JF (2002) Nation-wide survey on radiation doses in diagnostic and interventional radiology in Switzerland in 1998. Health Phys 83:46-55

5. Aroua A, Decka I, Burnand B, Vader JP, Valley JF (2002) Dosimetric aspects of a national survey of diagnostic and interventional radiology in Switzerland. Medical Phys 29:2247-2259
6. Galanski M, Nagel HD, Stamm G (2007) Paediatric CT exposure practice in the federal republic of Germany: results of a nationwide survey in 2005-2006. Medizinische Hochschule, Hannover

7. Kanae N, Masaki M, Kazuo I, Takashi M (2004) Survey of CT practice in Japan and collective effective dose estimation. Nippon Acta Radiologica 64:151-158

8. Nationwide Evaluation of X-Ray Trends (NEXT): 2000 Survey of Computed Tomography (2006), CRCPD publication NEXT 2000CT-T

9. Donnelly LF, Emery KH, Brody AS, Laor T, Gylys-Morin VM, Anton CG, Thomas SR, Frush DP (2001) Minimizing radiation dose for paediatric body applications of single-detector helical CT: strategies at a large children's hospital. AJR Am J Roentgenol 176:303-306

10. Cody DD, Moxley DM, Krugh KT, O'Daniel JC, Wagner LK, Eftekhari F (2004) Strategies for formulating appropriate MDCT techniques when imaging the chest, abdomen, and pelvis in pediatric patients. AJR Am J Roentgenol 182:849-859
11. Hollingsworth C, Frush DP, Cross M, Lucaya J (2003) Helical CT of the body: a survey of techniques used for pediatric patients. AJR Am J Roentgenol 180:401-406

12. Vock P (2005) CT dose reduction in children. Eur Radiol 15:2330-2340

13. Boone JM, Geraghty EM, Sielbert JA, Wootton-George SL (2003) Dose reduction in pediatric CT: a rational approach. Radiology 228:352-360

14. Paterson A, Frush DP, Donnelly LF (2001) Helical CT of the body: are settings adjusted for pediatric patients? AJR Am J Roentgenol 176:297-301

15. Huda W, Lieberman KA, Chang J, Roskopf ML (2004) Patient size and xray technique factors in head computed tomography examinations. II. Image quality. Med Phys 31:595-601

16. Siegel MJ, Schmidt B, Bradley D, Suess C, Hildebolt C (2004) Radiation dose and image quality in pediatric CT: effect of technical factors and phantom size and shape. Radiology 233:515522 
17. Verdun FR, Lepori D, Monnin P, Valley JF, Schnyder P, Gudinchet F (2004) Management of patient dose and image noise in routine pediatric CT abdominal examinations. Eur Radiol 14:835-841

18. Wilting JE, Zwartkruis A, van Leeuwen MS, Timmer J, Kamphuis AG,

Feldberg M (2001) A rational approach to dose reduction in CT: individualized scan protocols. Eur Radiol 11:26272632

19. Pages J, Buls N, Osteuax M (2003) CT doses in children: a multicentre study. Br J Radiol 76:803-811

20. Linton OA, Mettler FA (2003) National conference on dose reduction in CT with an emphasis on pediatric patients. AJR Am J Roentgenol 181:321-329

21. Hall EJ (2002) Lessons we have learned from our children: cancer risks from diagnostic radiology. Pediatr Radiol 32:700-706
22. Ron E (2002) Let's not relive the past: a review of cancer risk after diagnostic or therapeutic irradiation. Pediatric Radiology 32:739-744 discussion 751-754

23. Brenner DJ, Elliston CD, Hall EJ, Berdon WE (2001) Estimated risks of radiation-induced fatal cancer from paediatric CT. AJR Am J Roentgenol 176:289-296

24. Frush DP, Donnelly LF, Rosen NS (2003) Computed tomography and radiation risks: what pediatric health care providers should know. Pediatrics 112:951-957

25. International Commission on Radiological Protection (1997) Radiological protection and safety in medicine. ICRP Publication 73, Pergamon, Oxford New York

26. Schrimpton PC, Hillier MC, Lewis MA, Dunn M (2005) Dose from CT examination in the UK-2003 review. NRPB-W67 Report. National Radiological Protection Board, Chilton
27. International Electrotechnical Committee (2002) Medical diagnostic X-ray equipment-Particular requirements for the safety of X-ray equipment for CT; Standard IEC \#60601-2-44

28. http://www.impactscan.org

29. Neofotistou V (2001) Review of patient dosimetry in cardiology. Radiat Prot Dosim 94:177-182

30. Aroua A, Besançon A, BuchillierDecka I, Trueb P, Valley JF, Verdun FR, Zeller W (2004) Adult reference levels in diagnostic and interventional radiology for temporary use in Switzerland. Radiat Prot Dosim 111:289-295

31. Shrimpton PC, Wall BF (2000) Reference doses for paediatric computed tomography. Radiat Prot Dosim 90:249-252

32. Martin DR, Semelka RC (2006) Health effects of ionising radiation from diagnostic CT. Lancet 367:1712-1714 